\title{
Design of Energy Efficient Protocol for Homogeneous Mobile Wireless Sensor Network
}

\author{
Himani Verma ${ }^{1}$ and Madhu Priya ${ }^{2}$ \\ Student, Gautam Buddha University, Greater Noida, India ${ }^{1,2}$
}

\begin{abstract}
This paper brings clustering protocol which divides the whole network to variable radiuses with virtual circles where radiuses of each virtual circles and the cluster size will increase with the increase in distance from the base station. In this protocol leader node is introduced which is responsible for collecting and compressing data in each cluster from their ordinary neighbor node and sending them to head cluster. Simulation result demonstrate that proposed protocol in comparison with protocol such as LEACH, DSBCA and TCAC increases the network lifetime and makes network energy efficient across the network.
\end{abstract}

Keywords: LEACH, DSBCA, TCAC, Wireless Sensor Network, Protocols.

\section{INTRODUCTION}

Advancement in hardware technology provides small sensors with limited energy, computing power and wireless communication capability. Nodes placed in the network senses various data from the network and perform processing after that send message to the base station directly or in multi hop fashion.WSN can be deployed in the circumstances such as military, healthcare [1-3] natural disaster where human cannot withstand affectively .But the major drawback of sensors is that they have limited memory, low power \& energy constrained due to their small size. Wireless network can be deployed in extreme environment conditions. Sensor needs battery power for the operation such as data collection, data evaluation and to communicate with one another. When the power vanished, the performance of sensor completely stop. Due to this some information loss or sometime whole communication stops if that sensor is head node. So one of the main concern in the WSN is energy because in some cases unsafe area there is no possibility of charging or recharging the battery of the sensor [4 5].

So our main concern is how to make wireless sensor network energy efficient for that may technique are given which are data aggregation minimizing control packets and topology control integration are provided. Data aggregation helps in rising network lifetime by decreasing resources consumption. In this process combining of similar data is done which are from the same event which reduces battery power \& bandwidth. Routing mechanism has large share in energy consumption so it should be efficiently used [6-8]. The challenging issues in WSN are routing, in which many studies are also done. For increasing the lifetime clustering is also a major technique which reduces the energy consumption by all the nodes in the network [9-10]. In the hierarchical routing nodes are elected as cluster head which perform data collection and data aggregation. This technique save energy as it reduces intra cluster communication as distance between cluster head and cluster nodes reduces due to which the amount of data deliver to base station is reduced[11].Hierarchical routing in which cluster are formed are the best routing technique in which the whole network is divided in clusters in which one node is elected as cluster which collects the data from the ordinary node and perform data aggregation and send data to the base station which provide batter communication in topology management [1215]. In the homogeneous network all the sensor node are same in terms of energy and resources. Member of any cluster may be fixed or variable that depends on the protocol which is used in the network.

In this paper, the new energy efficient protocol is proposed based on leader node as the whole network is divided into virtual circles which helps in balancing of energy in the network. In this algorithm in each cluster one cluster head and leader node is elected that are responsible for collecting and compressing of data from ordinary nodes in the cluster. Leader node collects data from the ordinary node and performs data aggregation and then send data to cluster head and data is forwarded to base station in multi hop view.

\section{RELATED WORK}

In recent years, many networks based on clustering are proposed saving of energy is the critical concern in designing the wireless sensor network with clustering. Arrangement of nodes, architecture, characteristics operation and method that have been developed are different for different protocol.

LEACH is the most famous \& first energy efficient protocol with adaptive clustering of nodes. In this protocol each node assigned with some probability \& it's used to become head of cluster for each data transmission round \& to become cluster head is rotated among cluster member. In this protocol single hop routing is used [19] and in the 
network sensor node is uniformly distributed. Every node generates a random number and comprising it with threshold value which decides that node is cluster head in this round or not. Disadvantage of this protocol is that possibility of low energy node to be a cluster head \& longevity in the large scale network.

The power efficient gathering in sensor information system (PEGASIS) [23] protocol is not the clustering protocol but this protocol reduces the size of data sent to base station by compression \& remove redundant information. At the beginning of each round chain is formed \& process of formation of chain is centralized \& decentralized approach.

In HEED the sensor node based on probability to hybrid of energy are provided to each node based on that only sensor node with higher residual energy \& also decreases intra communication cost become the cluster head[24] which is basically a multi level energy feature to elect cluster head in this multi hop routing protocol is used

An energy efficient clustering scheme in wireless sensor networks (EECS)[25] protocol candidate of cluster head having constant \& specific radius start compete with other candidate \& node with the highest remaining energy will be elected as the cluster head. This protocol overcomes the drawback of LEACH protocol .But the drawback of this protocol is the increase in data overhead.

Unequal cluster based routing protocol (UCR) [26] is a hierarchical based routing protocol. Cluster head selection process is same as EECS protocol but the radiuses of node is variable so that nodes always from the main centre have larger radiuses which is due to cluster size nearer to the centre be small so that less energy in inter cluster communication is utilized which helps in energy consumption in the network .

In the topology controlled adaptive clustering (TCAC) [27] protocol is the balances the load on the cluster head by proper distribution of the cluster head .It works in decentralized way. First of all ,random nodes is elected as cluster head candidate \& than as the EECS protocol elected as cluster head .Secondly, cluster head send hello message to all other node in the network .After this stage. Ordinary node using prioritize table choose their cluster head. After this, cluster head recognize their number. In this protocol data is send to BS in single hop process. Drawback of this protocol is lots of data overhead \& each node has to send data twice to its cluster head.

Energy aware clustering algorithm with non uniform distributed node (EADC) uses an energy aware algorithm. It consists of three phases: Information collection phase, in this process each node sends their information to the other node through its IDs \& informs about its remaining energy with a fixed radius. In the next phase, cluster head competition is done. In which any node which fails to send the head message sends in a certain interval. In the last phase, cluster information is done. Each ordinary node selects cluster head to sends its data which is based on the residual energy of the node \& average residual energy to the neighboring node. In the sending of data to the BS, if the distance is less than the threshold distance which protocol parameter then it sends data directly otherwise in a multi hop fashion. In local energy consumption prediction based clustering protocol (LECP-CP) [35] in which cluster head is elected based on the local energy consumption prediction. Routing tree is used to done inter cluster communication in the network but intra- cluster communication is performed directly. If the distance of node from the BS is less than the threshold distance than it sends its data directly to the BS otherwise through multi-hop routing tree \& this phase is known as data transmission.

\section{PROTOCOL DETAILS}

The main objective of this paper is to design energy efficient protocol for homogeneous wireless sensor network which is based on the cluster head \& leader node which balances the load in the network \& validate the result with the existence benchmarks. In this protocol each node are provided with some responsibility according to their position in the network. In this protocol node with proper positioning \& maximum residual energy is elected as cluster head which is changed cyclically in each round. In this protocol there are two phases set up phase and steady phase

\subsection{Network model $\&$ assumption:}

All the assumptions of the proposed protocol are as follows:

- Nodes deployed in the network are random

- All the nodes are homogeneous and they are distributed uniformly.

- All the nodes are same in the resource \& initial energy.

- Base station is located at the center of the network

- All the nodes are dynamic in the nature and do not have GPS.

The model which is used in the protocol is simplified model [19] for the energy consumption in communication when a node sends $\mathrm{k}$ bits of packet which is as follows:

$$
E_{\mathrm{s}}= \begin{cases}k *\left(E_{\mathrm{elec}}+E_{\mathrm{fs}} * d^{2}\right) ; & d<d_{0} \\ k *\left(E_{\mathrm{elec}}+E_{\mathrm{Mp}} * d^{4}\right) ; & d \geq d_{0}\end{cases}
$$


If distance $d \leq$ do than free space model is used otherwise multi path fading channel is used.

The consumption of energy when nodes received packet $\mathrm{k}$ is as follows:

$$
E_{R}=k * E_{\text {elec }}
$$

Data aggregation energy will be computed as

Eagg $=$ Energy required for aggregation a bit $* \mathrm{~K} *$ no. of message

\subsection{Set Up Phase:}

The set up phase is divided into three phase, first phase is cluster head selection, selection of leader node \& next hop selection phase is the last phase.

\subsubsection{Phase 1: Election of cluster head}

Protocol makes distribution of cluster head efficiently. Balancing of cluster head is also done through suitable distribution of cluster head which also helps in decrease in intra cluster communication. The main criteria of protocol are to choose the suitable node for cluster head so that increase in the energy efficient of the network \& lifetime of the network increases. By using Euclidean distance algorithm every node have the distance estimation of all the other node.

Steps of this process are as follows:

- base station broadcast HELLO MESSAGE to all the network in the node with its ID

- All the nodes estimate there distance to BS according to received signal strength.

- all the node include its ID broadcast initial message to all the network with a initial message

After this all the node calculates the cluster head $\mathrm{R} \mathrm{CH}$ using the following equation:

$$
R_{\mathrm{CH}}=R_{\min } *\left[1+\left(\frac{d_{\mathrm{BS}}-d_{\mathrm{BS} \min }}{d_{\mathrm{BS} \max }-d_{\mathrm{BSmin}}}\right)\right]
$$

Rmin is the size of cluster which is minimum size \& it is taken as parameter for the protocol dBSmin is the distance in between the nearest node \& base station dBSmax is the farthest node distance \& the base station. After this each node calculate the value.

$$
F_{\mathrm{CH}-\text { value }}=\alpha * N_{\mathrm{deg}}+\frac{\beta}{\mathrm{MSD}_{\mathrm{deg}}}+\frac{\gamma}{d_{\mathrm{BS}}}
$$

$\alpha, \beta, \gamma$ are constant between $0 \& 1$ whoes sum is 1 ,Ndeg is the amount of neignbouring node with RCH radius , MSDdeg is the mean square of distance between neighbouring node

After the calculating of $\mathrm{MCH}$-value each node generate random number between $0 \& 1$. If the random value generated in less than MCH-value than that node consider itself as the volunteer cluster head candidate. After this all the cluster head candidate based on criteria of $\mathrm{RCH}$ radius \& residual energy elected as cluster head \& than message all other nodes in the network with its IDs as cluster head \& all neighbour node determines its cluster head IDs based on received signal strength.

\subsubsection{Phase 2 : Election of leader node}

In the protocol only cluster head are elected to collect data from the normal node but in this protocol leader node is also introduced to take data from the node \& data aggregation is done in large amount which also decreases the distance of communication in the network by which nodes density also aggeft the intra cluster energy. To choose proper node for the leader node, all the ordinary node calculate MLN value.

$$
F_{\mathrm{LN}-\text { value }}=\eta * M_{\mathrm{deg}}+\frac{\lambda}{K_{\mathrm{LN}}}
$$

Mdeg is the number of neigbouring node within leader radius, KLN is the number of nodes for leader node .

$\eta$ and $\lambda$ are the constant weight between 0 and 1 .

Node with heigher residual energy based on MLN value will be elected as leader node \& broadcast its ID in the network \& all the node based on its signal strength estimate its distance from that node. 


\subsubsection{Phase 3: Next Hop selection}

If the node is the ordinary node that each node consider its cluster as next destination to transfer data. If the distance between the cluster head is larger than the leader node than next hop is leader node otherwise to cluster head.If the node is cluster head than its next hop will be the nearest head. Transmission of message in its protocol is done in TDMA program.

\subsection{Steady state phase:}

In this protocol data aggregation is done in all the cluster head \& leader node .After that they will transfer aggregate data to base station in a multi hop fashion.

\section{SIMULATION RESULTS}

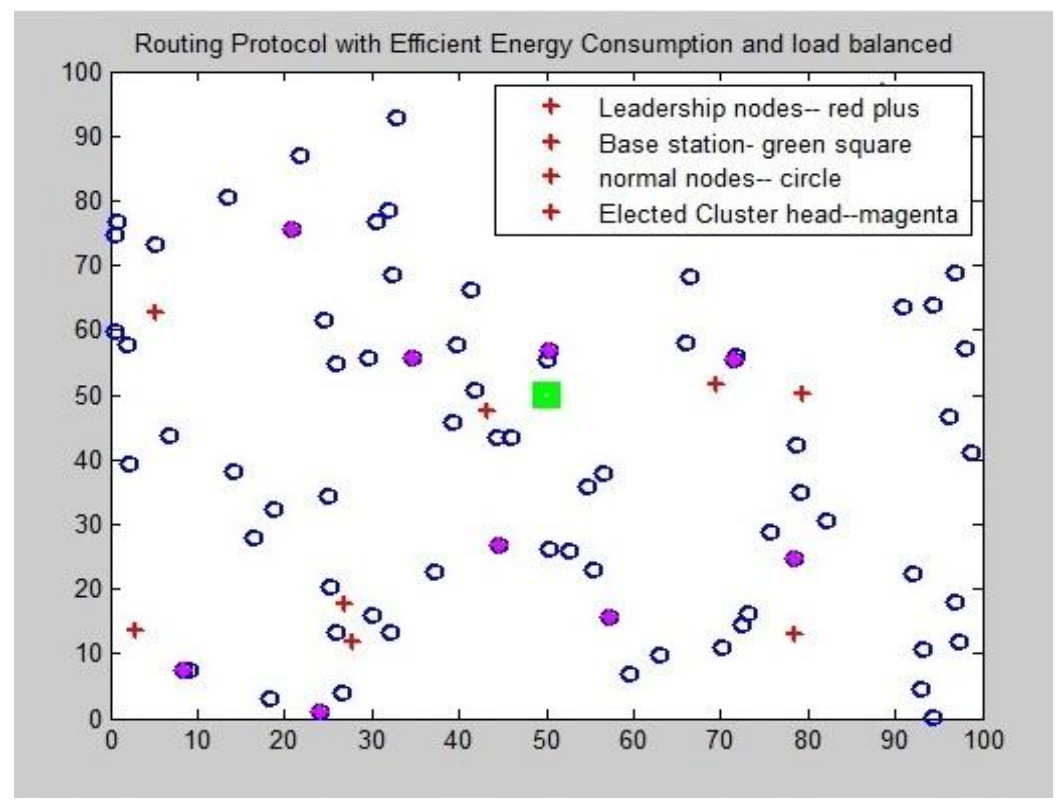

Figure 1 Multilayer structure of scenario of network

In this paper main target is to achieve energy efficient protocol.the main criteria used to determine the protocol is the lifetime of the network. The lifetime is the time when network starts for work to a round when the first node to release energy and is called resistant to change period.

All the simulation and model are prepared and conducted in the MATLAB software and the placement of nodes is completely random. Simulation parameters of the proposed protocol are as follows:

- area of the network is taken as $(0,0)-(100,100)$

- The base station is located at the center of the network, number of nodes in the network is 100

- The Initial energy of all nodes is $0.5 \mathrm{~J}$

- Free space channel model (Efs) is $50 \mathrm{nj} / \mathrm{bits}$

- multi path fading channel model (Emp) is $0.0013 \mathrm{pj} / \mathrm{bits} / \mathrm{m}$

- distance threshold (do) $87 \mathrm{~m}$

- Data aggregation energy (Eda ) $5 \mathrm{Nj} / \mathrm{bits} / \mathrm{signal}$

- DP size data packet size in bit is 4000

- CP size control packet size in bit is 200

Proposed protocol is evaluated and compared with the bencgmark protocol such as LEACH ,TCAC, and a balanced clustering algorithm with distributed self organisation for wireless sensor network (DSBCA) based on important criteria such as performance of packet deliver to BS,Performance of packet deliver to cluster head, energy consuption, remaining energy, performance of node alive in the WSN, Performance of selection of cluster head. According to the comparision the technique proposed in this paper is scalable and can improve energy efficiency in the network with different sizes.

Figure 2 is the data bits transmitted in the network which is shown in the figure 1.last bit is the parity bit which is used to determine the error detection process. Figure 3 is the error detection at the receiver in the proposed model of the network 


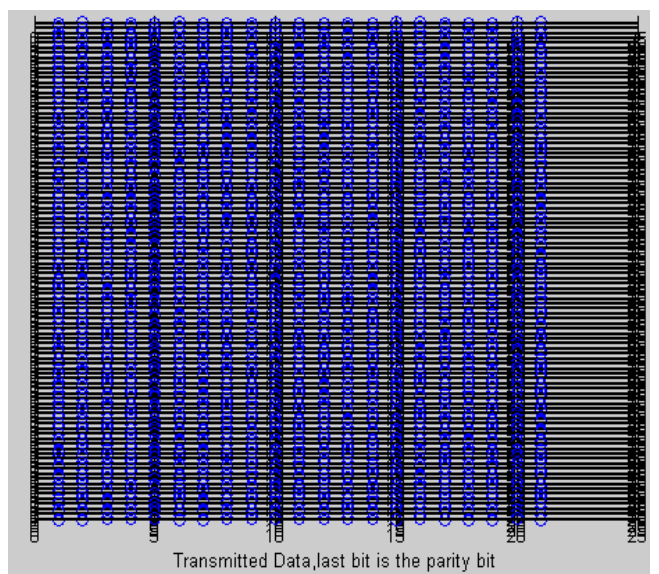

Figure 2 Transmitted data bits in the network

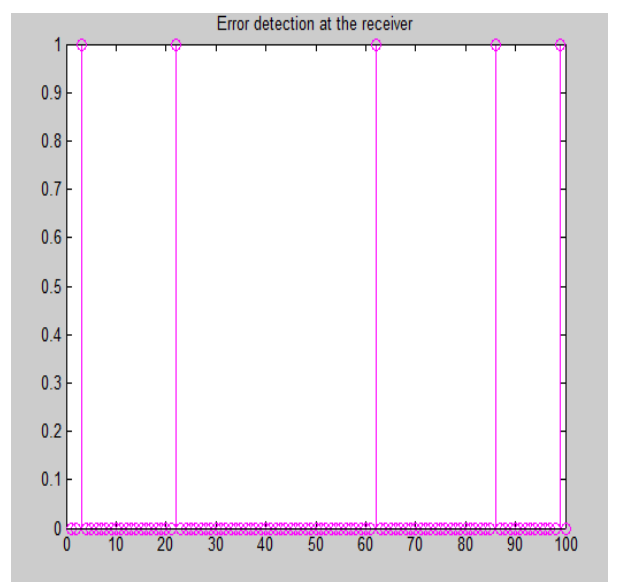

Figure 3 Error detection at the receiver

As in the figure 4 the performance of node alive in the network is determined and is compared with the protocol such as LEACH , TCAC , DSBCA. The value suggest that the proposed protocol has extended the number of alive node in the network with more round and enhance the network performance. Moreover it is clear from the figure that instability period for proposed network is also improved in the network which is the death of the first node to the last node .It also clarifies that the proposed protocol has better distribution and network coverage .

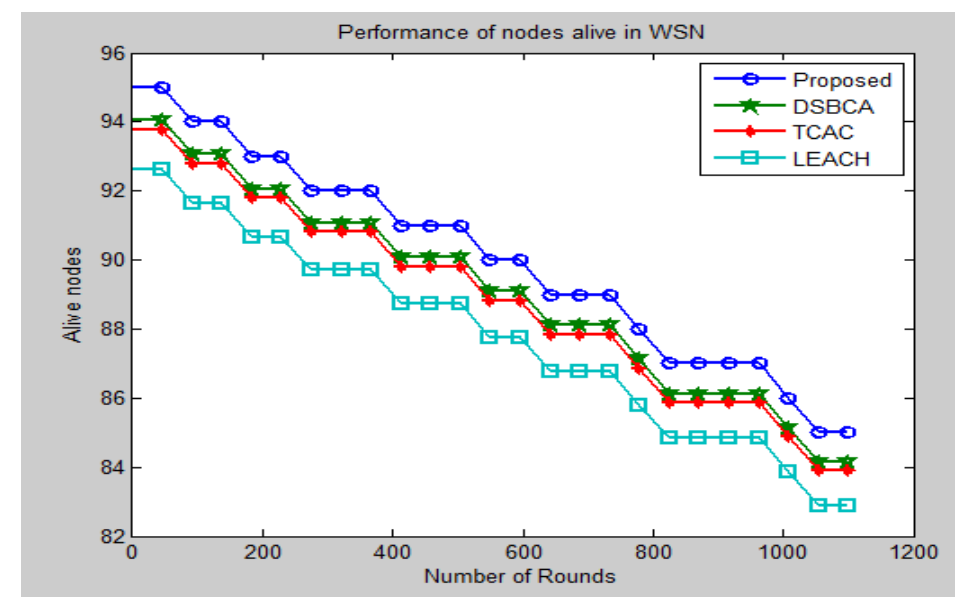

Figure 4: Performance of nodes alive in WSN

Figure 5 shows the performance of selection of cluster head shows that as the number of rounds increases selected cluster head count decreases as radius of each virtual circle and the size of each cluster will increase with increase with the increasing distance from the base station.

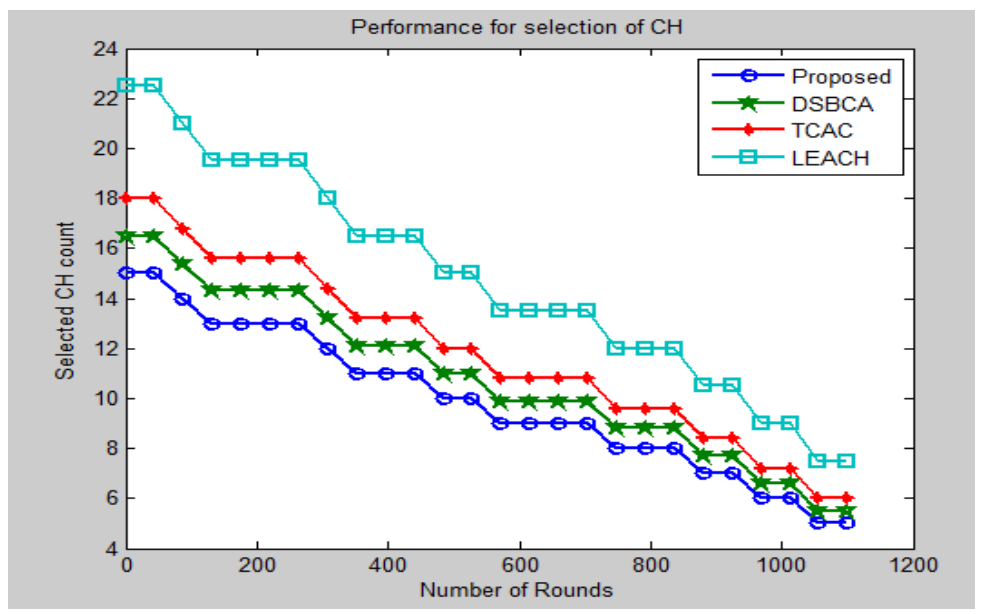

Figure 5 performance of selection of cluster head 
Figure 6 shows the total number of packets received by BS by the proposed protocol in comparison with LEACH, TCAC, and DSBCA protocol. the results depicts that performance is same for all the protocol but it increases when the number of rounds increase that means with increase of round the packet deliver to BS increases with the proposed protocol which reflects the best performance of proposed protocol

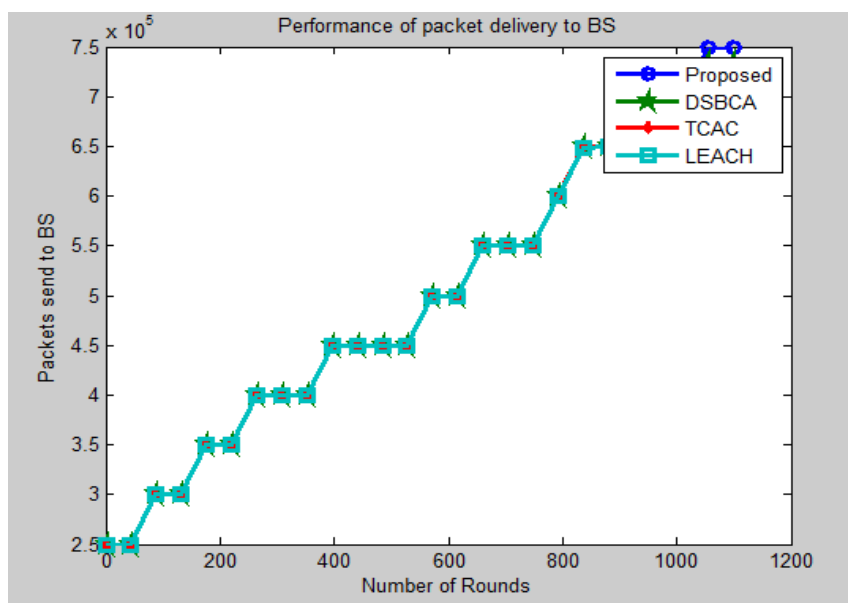

Figure 6: Performance of packet deliver to BS

Figure 7 shows the performance of packet deliver to cluster head is determined by the number of packet deliver to cluster head in each round which is also increases as the number of round increases in the network.

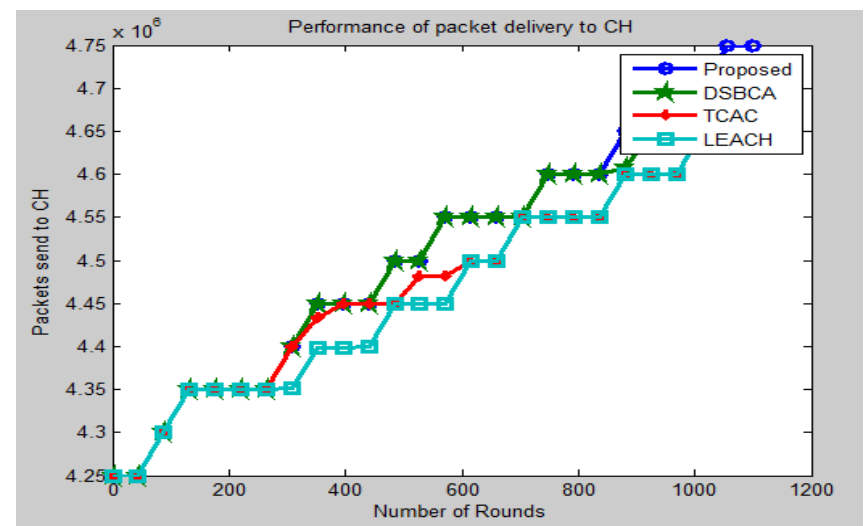

Figure 7: performance of packet deliver to $\mathrm{CH}$

Figure 8 shows that energy consumption the reduces in comparison to the other protocol and it shows that energy consumption and immediate changes of energy consumption per round for proposed protocol is much less than LEACH , TCAC and DSBCA protocol that demonstrates the balanced and uniform energy consumption throughout the network of proposed protocol

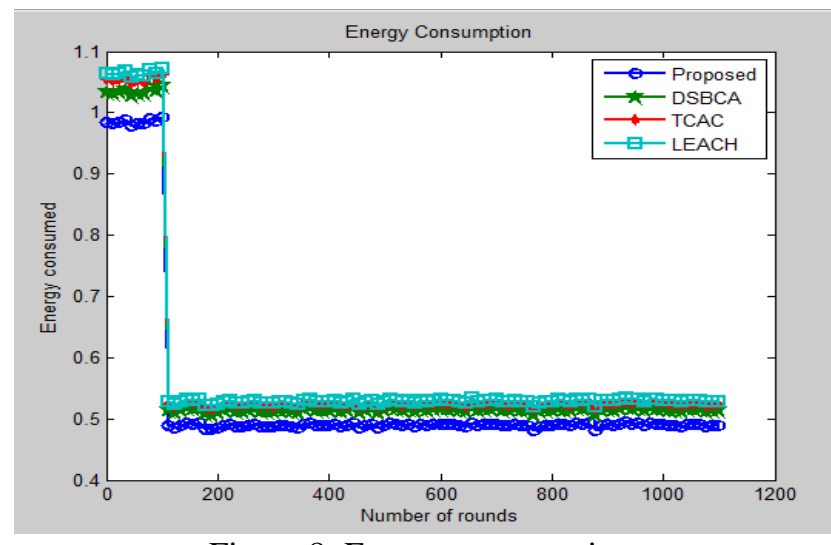

Figure 8: Energy consumption 


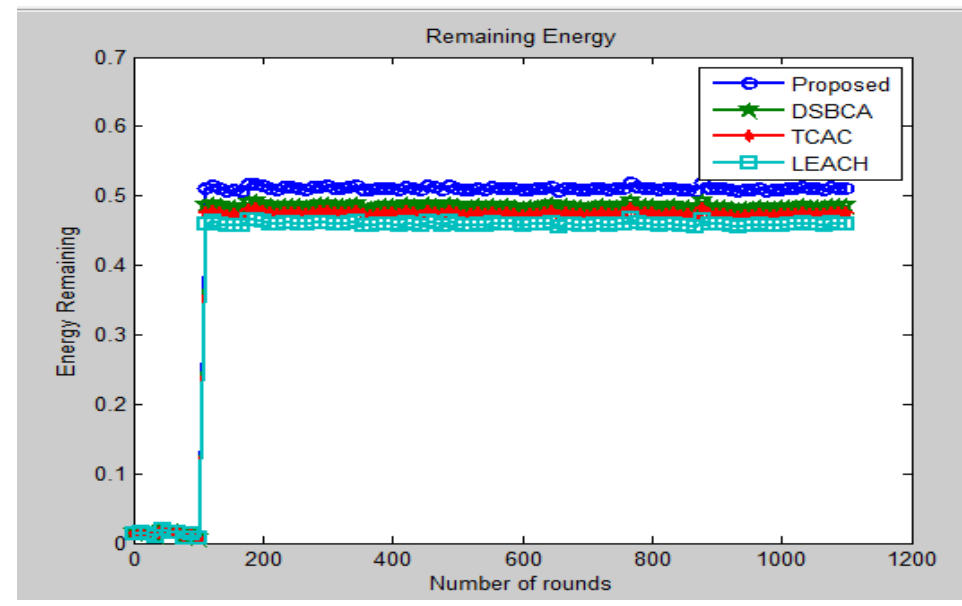

Figure 9 shows the remaining energy in the network. It is the residual energy in the network at the end of each round for proposed protocol is more than LEACH, TCAC and DSBCA protocols this describes the best improved performance of proposed protocol in terms of reducing energy consumption per round. Moreover, the percentage of residual energy after the death of first node is also reduces

\section{CONCLUSIONS}

In this paper, energy saving protocol was instigate that utilizes essential circles that form multilayer circular structure across the network and innovative method for intra cluster communication which balances the distribution of energy utilization across the network and also causing load balancing, proposed protocol increased network lifetime notably in such a way that the lifetime of network increased with the proposed protocol.

\section{REFERENCES}

[1] Akyildiz, I.F., Su, W., Sankarasubramaniam, Y., et al.: 'A survey on sensor networks', IEEE Commune. Mag., 2002, 40, (8), pp. 102-114

[2] Akyildiz, I.F., Kasimoglu, I.: 'Wireless sensor and actor networks: research challenge', Ad Hoc Netw., 2004, 2, (4), pp. 351-367

[3] Pantazis, N.A., Nikolidakis, S.A., Vergados, D.: 'Energy-efficient routing in wireless sensor networks', IEEE Commun. Serv. Tutor., 2013, 15, (2), pp. 551-592

[4] Sungjin, A., Daeyoung, K.: 'Proactive context-aware sensor networks'. Third European Workshop, Zurich, Switzerland, 2006, pp. 38-53

[5] Perillo, M., Cheng, Z., Heinzelman, W.B.: 'On the problem of unbalanced load distribution in wireless sensor networks'. IEEE GLOBECOM Wireless Ad Hoc And Sensor Networks, 2004, pp. 74-79

[6] Premita, A., Katiyar, M.: 'A review on power efficient energy aware routing in wireless sensor networks', Int. J. Eng. Res. Technol., 2012, 1, (4), pp. 1-8

[7] Yick, J., Mukherjee, B., Ghosal, D.: 'Wireless sensor network survey', Comput. Netw., 2008, 52, (12), pp. 2292-2330

[8] Ozdemir, S., Xiao, Y.: 'Secure data aggregation in wireless sensor networks: a comprehensive overview', Comput. Netw. 2009, 53, (12), pp. 2022-2037

[9] Manfredi, S.: 'Reliable and energy-efficient cooperative routing algorithm for wireless monitoring systems', IET Wirel. Sens. Syst., 2012, 2, (2), pp. 128-135

[10] Rizk, R., Elhadidy, H., Nassar, H.: 'Optimized mobile radio aware routing algorithm for wireless sensor networks', IET Wirel. Sens. Syst., 2011, 1, (4), pp. 206-217

[11] Hoang, D.C., Kumar, R., Panada, S.k.: 'Realisation of a cluster-based protocol using fuzzy C-means algorithm for wireless sensor networks', IET Wirel. Sens. Syst., 2013, 3, (3), pp. 163-171

[12] Singh, S.G., Singh, M.P., Singh, D.K.: 'A survey on energy efficient hierarchical cluster-based routing in wireless sensor networks', Int. J. Adv. Netw. Appl., 2010, 2, (2), pp. 570-580

[13] Tarhani, M., Kavian, Y.S., Siavoshi, S.: 'SEECH: scalable energy efficient clustering hierarchy protocol in wireless sensor networks', IEEE Sens. J., 2014, 14, (11), pp. 3944-3954

[14] Mahani, A., Kargar, A., Kavian, Y.S., et al.: 'Non-uniform distribution of multi-hop sensor networks: performance improvement and energy hole mitigation', IET Wirel. Sens. Syst., 2012, 2, (4), pp. 302-308

[15] Kumar, D.: 'Performance analysis of energy efficient clustering protocols for maximising lifetime of wireless sensor networks', IET Wirel. Sens. Syst., 2014, 4, (1), pp. 9-16

[16] Huang, P., Xiao, L., Soltani, S., et al.: 'The evaluation of MAC protocols for wireless sensor networks: a survey', IEEE Commun. Surv. Tutor., 2013, 15, (1), pp. 101-120

[17] Abbasi, A.A., Younis, M.: 'A survey on clusterings for wireless sensor networks', Comput. Commun., 2007, 30, (14), pp. 2826-2841

[18] Zairi, S., Zouari, B., Niel, E., et al.: 'Nodes self-scheduling approach for maximis wireless sensor network lifetime based on remaining energy', IET Wirel. Sens. Syst., 2012, 2, (1), pp. 52-62

[19] Heinzelman,W.B., Chandrakasan, A.P., Balakrishnan, H.: 'An application-specific protocol architecture for wireless microsensor networks', IEEE Trans. Wirel. Commun., 2002, 1, (4), pp. 660-670

[20] Kaur, T., Baek, J.: 'A strategic deployment and cluster-header selection for wireless sensor networks', IEEE Trans. Consum. Electron, 2009, $55,(4)$, pp. $1890-1897$

[21] Choe, J., Xu, J.: 'Energy balancing LEACH for wireless sensor networks', Int. J. Intell. Inf. Process., 2012, 3, (2), pp. 56-65 


\section{IJARCCE}

[22] Chen, H., Zhang, C., Zong, X., et al.: 'LEACH-G: an optimal cluster-heads selection algorithm based on LEACH', J. Softw., 2013, 8, (10), pp. 2660-2667

[23] Lindey, S., Raghavendra, C.S.: 'PEGASIS: power efficient gathering in sensor information system'. Proc. IEEE Aerospace Conf., 2002, vol. 3 , pp. $1125-1130$

[24] Younis, O., Fahmy, S.: 'HEED: a hybrid, energy-efficient, distributed clustering approach for ad hoc sensor networks', IEEE Trans. Mob. Comput., 2004, 3, (4), pp. 366-379

[25] Ye, M., Li, C., Chen, G., et al.: 'an energy efficient clustering scheme in wireless sensor networks', Ad Hoc Sens. Netw., 2006, 3, pp. 99-119

[26] Chen, J., Li, C., Ye, M., et al.: 'An unequal cluster based routing protocol in wireless sensor networks', Wirel. Netw., 2009, 15, (2), pp. 193207

[27] Dahani, D.P., Singh, Y.P., Ho, C.K.: 'Topology-controlled adaptive clustering for uniformity and increased lifetime in wireless sensor networks', IET Wirel. Sens. Syst., 2012, 2, (4), pp. 318-327

[28] Aslam, N., Phillips, W., Robertson, W., et al.: 'A multi-criterion optimization technique for energy efficient cluster formation in wireless sensor networks', Inf. Fusion, 2011, 12, (3), pp. 202-212

[29] Liu, T., Li, Q., Liang, P.: 'An energy-balancing clustering approach for gradient-based routing in wireless sensor networks', Comput. Commun., 2012, 35, (17), pp. 2150-2161

[30] Yu, J., Qi, Y., Wang, G., et al.: 'A cluster-based routing protocol for wireless sensor networks with nonuniform node distribution', AEÜ - Int. J. Electron. Commun., 2012, 66, (1), pp. 54-61

[31] Yu, J., Feng, L., Jia, L., et al.: 'A local energy consumption prediction-based clustering protocol for wireless sensor networks', Sensors, 2014, 14, (12), pp. 23017-23040

[32] Liu, Y., Xiong, N., Zhao, Y., et al.: 'Multi-layer clustering routing algorithm for wireless vehicular sensor networks', IET Commun., 2010, 4, (7), pp. 810-816

[33] Liao, Y., Qi, H., Li, W.: 'Load-balanced clustering algorithm with distributed self-organization for wireless sensor networks', IEEE Sens. J., 2013, 13, (5), pp. 1498-1506

\section{BIOGRAPHIES}

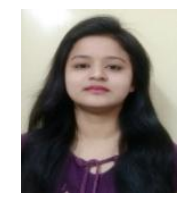

Himani Verma, presently pursuing M.TECH in wireless communication and networks from Gautam Buddha University, Greater Noida, Uttar Pradesh. Received B.TECH in 2014 from Vivekanand Institute of Technology and Science, Ghaziabad, Uttar Pradesh. Area of research interest are Wireless sensor networks, Mobile computing, Information Security, Optical system and smart antenna.

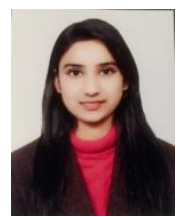

Madhu Priya, presently pursuing M.TECH in wireless communication and networks from Gautam Buddha University, Greater Noida, Uttar Pradesh. Received B.TECH in 2015 from Guru Jambheshwar University of Science and Technology, Hisar, Haryana. Area of research interest are Wireless sensor networks, Signal Processing, Computer Network, Information Security and smart antenna. 